\title{
Performance Measurements of Evacuation Systems
}

\author{
GUNNAR G. LøVÅS
}

Department of Mathematics, University of Oslo

P.O. Box 1053, N-0316 Oslo, Norway

\begin{abstract}
The paper dicusses how the use of performance based safety codes is closely related to the availability of reliable tools to evaluate system performance. Several performance measures are defined for an evacuation system, and the paper presents an example of a simulation program designed to evaluate these measures.
\end{abstract}

KEYWORDS: Fire Risk Assessment, Performance Based Codes, Human Behaviour, Evacuation Simulation.

\section{PERFORMANCE BASED SAFETY CODES}

In order to increase the fire safety level and reduce the risks as low as reasonable practicable, performance based codes have entered the scene. Performance codes establish safety goals and leave the means of achieving those goals to the designer. This is in contrast to the tradidional prescriptive codes that prescribe precisely how something is to be done in a given circumstance. Crucial to the practicability of performance codes is an objective method of evaluating the ability of the proposed design to meet the established goals. Equally important, the goals must be expressed in measurable terms. As an example, consider three possible requirements to an evacuation system: 
1. Qualitative Performance Goals (QPG), e.g. evacuation should be safe.

2. Quantitative Performance Requirements (QPR) on system level, e.g. the total evacuation time should be below $10 \mathrm{~min}$.

3. Detailed Specific Requirements (DSR) to "component solutions", e.g. doors should be wider than 1.2 meters.

Which of these statements are the most useful? In the future, probably, codes will be composed of all three types of statements. QPGs will be used to motivate the fire safety work, and QPRs will communicate what it means to achieve the goals. For certain elements of a system, DSRs will set a minimum standard. See Bukowski and Tanaka [1] for further details.

The main challenge for the fire safety community on it way towards performance based codes, is to arrive at reasonable solutions to QPRs. The rest of this paper is dedicated to this problem. Among many others, these questions must be answered: Which performance measures (indicators) are of relevance? At what value of a performance measure does one arrive at a QPG? What can be done to improve performance, if necessary? How does one calculate (estimate) the performance measures? Is it possible to believe in these calculations?

The field of fire safety science involves a lot of quite diverse activities. In order to keep the presentation at a reasonable practical level, and to stay within the field of the author, let us concentrate on the problem of building evacuation. The elements of the following discussions, and their basic ideas should be compatible to many other areas of fire safety.

\section{PERFORMANCE MEASURES}

Performance measures (PMs) of evacuation systems (ESs) describe the likely behavior of the evacuation process, i.e. how the ES is able to function in an emergency situation. Therefore, typical and important examples of such measures are:

- Expected number of fatalities.

- Expected COHb level in the evacuees' blood.

- Expected number of safe evacuees at time $t$.

- Expected time needed to evacuate $k$ persons.

The measures are related to a specific set of scenarios, describing the accident, the population distribution, etc. Some PMs will be precicely defined, based on a mathematical model of the evacuation process, following the basic lines in L $\varnothing$ vås [9]. 


\section{Model Parameters}

A building composed of $N$ rooms and $M$ doors will be modelled as the network $G(V, E)$, consisting of the nodes $v_{1}, \ldots, v_{N}$ in the set $V$ and $M$ links in the set $E$. We write $e_{i j}=\left(v_{i}, v_{j}\right) \in E$ if and only if there is a link between nodes $v_{i}$ and $v_{j}$. The nodes represent rooms, and the links represent doors. The nodes are divided into three subsets: Source nodes $V_{1}$, transportation nodes $V_{2}$ and exit (destination) nodes $V_{3}$. The nodes in $V_{3}$ are absorbing, i.e. persons will not move from a node in $V_{3}$ to a node in $V_{1} \cup V_{2}$.

The population of finite size $K$ is modeled as $K$ separate individuals moving around in the evacuation network $G$, from nodes in $V_{1}$ to nodes in $V_{3}$. Their movement is influenced by human decisions (initial response and route choice logic) and walking ability. Many parameters may be used to describe the persons and their behaviour, but it is outside the scope of this paper to present them here. The persons are located in the building, at time $t=0$, with probability $p_{s}$, according to the rule $\underline{K}_{s}=\left(K_{1 s}, \ldots, K_{N_{s}}\right)$, where $K_{i s}$ is the initial number of persons in node $i$. The index $s$ is a member in the set $\mathcal{S}$ of indices for all possible location rules.

We syncronize our clocks so that at time $t=0$, the hazardous situation starts, e.g. when a flammable gas is ignited (syncronization is important, but the choice of timeorigin may be redefined). Assume that it is possible to define a reasonable time-horizon, $T \in(0, \infty)$, for the evacuation process, so that there is high probability that the process has terminated before $T$. We study the evacuation process in the time-interval $[0, T]$ only.

The accident (or hazard) is represented as a set of possible scenarios. The accident follows scenario $\underline{H}_{m}(t)=\left(H_{1 m}(t), \ldots, H_{N m}(t)\right)$ with probability $q_{m}$, in which case the accident effects in node $i$ at time $t$ can be expressed as $H_{i m}(t)$. For the index $m$ we have $m \in \mathcal{M}$, where $\mathcal{M}$ is the set of indices for all possible accident scenarios. With inspiration from multistate reliability theory, we require that $H_{i m}(t) \in\left\{0,1,2, \ldots, M_{H}\right\}$, where $H_{i m}(t)=0$ iff no accident effects are present, and $H_{i m}(t)=M_{H}$ iff the accident will kill anyone present in node $i$ at time $t$.

\section{Stochastic Model Variables}

The number of persons in node $i$ at time $t$ will be noted $X_{i}(t)$. Let the aggregate system state $\underline{X}(t)=\left(X_{1}(t), \ldots, X_{N}(t)\right)$ be an element in the finite space $\mathcal{X}$ of all possible system states.

Let $\underline{H}(t)=\left(H_{1}(t), \ldots, H_{N}(t)\right)$ describe the state of the accident. From the set of all possible accident scenarios, a specific scenario is "drawn" according to the probabilities $q_{m}$; if scenario $m$ is selected, then $\underline{H}(t)=\underline{H}_{m}(t)$.

A person will be exposed to accident effects depending on the route he follows and the length of time he spends in the different nodes. Let $A_{k}(t)$ be a vector describing 
the doses of accident exposure received by person $k$ at time $t$. Similarly, let $K_{k}(t)$ be an indicator taking value 1 if person $k$ is alive at time $t$ and value zero otherwise. Define $K(t)=\sum_{k} K_{k}(t)$ as the total number of persons being alive.

Define $M(t)$ as the number of persons who managed to evacuate within time $t$,

$M(t)=\sum_{i: v_{i} \in V_{3}} X_{i}(t)$.

The evacuation time for one person is defined as the total time he needs to get out of the building (i.e. to arrive at a node in $V_{3}$ ), including reaction time, walking time and waiting time in queues. The individual evacuation time of exiting person number $k$ is $T_{k}$. We define $T_{k}=T$ if person $k$ does not evacuate before $T$. Hence, the times are ordered so that $T_{1} \leq T_{2} \leq \cdots \leq T_{K}$

Node $i$ is busy for $Y_{i}$ time units, and the total time spent in node $i$ is $I_{i}$, hence defined as

$Y_{i}=\int_{0}^{T} I\left(X_{i}(t) \geq 0\right) d t$

$I_{i}=\int_{0}^{T} X_{i}(t) d t$

where $I(\cdot)$ equals one if $(\cdot)$ is true, zero otherwise. The last time when any persons are present in node $i$ is $L_{i}=\sup \left\{t \in[0, T]: X_{i}(t) \geq 0\right\}$. Let $L=\max L_{i}$, the maximalization taken over all nodes in $V_{1} \cup V_{2}$.

We define the average population size $\bar{X}_{i}(\gamma)$ of node $i$ as $\bar{X}_{i}(\gamma)=I_{i} / \gamma$, where $\gamma$ equals $Y_{i}, L_{i}$ or $L$, corresponding to three different types of averages. The maximum population size is $X_{i}^{\max }=\sup _{t} X_{i}(t)$.

Define $V_{3}^{\prime}$ as all nodes in $V_{1} \cup V_{2}$ which are neighbours to a node in $V_{3}$. A $v_{i} V_{3}^{\prime}$-path is a sequence of neighbouring nodes from $v_{i}$ to a node in $V_{3}^{\prime}$. A $v_{i} V_{3}^{\prime}$-path is safe at level $s$ at time $t$ iff $H_{j}(t) \leq s$ for all nodes $v_{j}$ in the path (in particular, a path is safe if it is safe at level 0 ). Two $v_{i} V_{3}^{\prime}$-paths are independent iff all their common nodes are in $v_{i} \cup V_{3}^{\prime}$.

Let $f_{i}(t, s)$ be the number of independent $v_{i} V_{3}^{\prime}$-paths that are safe at level $s$ at time $t$. The average redundancy number $F(t, s)$ is defined as

$$
F(t, s)=\frac{\sum_{i: v_{i} \in V_{1} \cup V_{2}} X_{i}(t) f_{i}(t, s)}{K-M(t)} .
$$




\section{Definition and Discussion}

It is necessary that we consider the whole range of possible values of the variables we have defined. Therefore, we will typically define the relevant PMs as the expected value of a stochastic variable, its distribution, quantiles, etc. Since many elements in a complex ES are subject to random fluctuations, it is often a problem that the stochastic variables, e.g. $T_{k}$ or $M(t)$, have very high variances, reducing the "information value" of the PMs. One way to overcome this problem is to measure the performance of the ES under more specificly given "initial conditions", e.g. that a specific population localization $K_{\text {s }}$ and an accident scenario $\underline{H}_{m}$ is known. The PMs may be studied for all interesting combinations of $s \in \mathcal{S}$ and $m \in \mathcal{M}$. In this way it is possible to identify the "critical components" in the ES, whether it is the accident development, the initial population distribution or the evacuation movement process itself.

The PMs are listed below in five categories, named $\Phi_{1 a}, \Phi_{1 b}, \ldots, \Phi_{2 a}, \Phi_{2 b}, \ldots$, A short discussion about the usefulness of the different measures is included.

Category 1. These measures are related to the effects the accident has on the population.

$\Phi_{1 a}=$ Prob. distr. of $K(t)$

$\Phi_{1 b}=E K(t)$

The safety of the building population is our main concern. Therefore, measures $\Phi_{1}$. are two of the most important measures, especially when evaluated at $t=T$. However, measure $\Phi_{1 b}$ will often be a poor measure since the expected value is not a good measure of centrality for a skew distribution. Using $\Phi_{1 a}$ to calculate the probability that more than $k$ persons are dead, is much more informative.

In practice, the measures $\Phi_{1}$. are used as "filters" in the sense that their values give an idea whether the ES performs bad or well. Even if the ES seems to perform well, one should study the other performance measures to see if the performance is really OK, or if it could easily be improved. This is in line with the idea that the risk for the building occupants should be "as low as reasonable practicable" (ALARP), an idea influencing much of modern risk analysis. time $t$.

Category 2. These measures are related to $M(t)$, the number of safe evacuees at

$$
\begin{aligned}
& \Phi_{2 a}=\text { Prob. distr. of } M(t) \\
& \Phi_{2 b}=E M(t) \\
& \Phi_{2 c}=\text { Percentiles of } M(t)
\end{aligned}
$$

Measures related to time are very important because most hazards evolve over time so that there is limited time available for safe escape. Let $T_{A}$ be the time available to escape the building. Obviously, $T_{A}$ is a stochastic variable, and its distribution will not be known to the building occupants (or anyone else). Nevertheless, based on knowledge about accidents and 
their potential development, it is customary to determine $T_{A}$ as a deterministic parameter. It is also possible to interpret $T_{A}$ as the time accepted by management, e.g. one QPR may be that $98 \%$ of the population should have evacuated within time $T_{\boldsymbol{A}}$.

Measures $\Phi_{2}$. tell quite a lot about the behaviour of the ES, they are easy to interpret, and they are a good basis for an evaluation of the ES's efficiency. Measures $\Phi_{2 b}$ and $\Phi_{2 c}$ carry valuable information over the whole time-span $[0, T]$, especially in the first part of the interval, while $\Phi_{2 a}$ is the best measure for high $t$-values, e.g. $t=T_{A}$. Observe also that $E M(t) \rightarrow E K(t)$ as $t \rightarrow \infty$; hence, there is a strong connection between measures $\Phi_{1}$. and $\Phi_{2 . .}$

Category 3. These measures are related to the evacuation time $T_{k}$.

$\Phi_{3 a}=$ Prob. distr. of $T_{k}$

$\Phi_{3 b}=E T_{k}$

$\Phi_{3 c}=$ A lower bound for $T_{K}$

The expected evacuation time $E T_{k}$ will often be a poor measure because:

- If $T$ is finite and $\operatorname{Pr}\left(T_{k}=T\right)>0$, then the evacuation process is truncated and $E T_{k}$ will be difficult to interpret.

- If $T=\infty$ and $\operatorname{Pr}\left(T_{k}<\infty\right)<1$, then we also get $E T_{k}=\infty$, which is not really very informative. This corresponds to an accident scenario where there is a positive probability that $(K-k+1)$ persons will be killed. Since there will always be a positive probability of some fatalities - otherwise it was not necessary to evacuate we know that $E T_{K}=\infty$.

The probability that all the $K$ persons have evacuated within the available time is an important number. This probability may be of interest at other times and other values of $k$ too, making $\Phi_{3 a}$ an interesting measure.

Measure $\Phi_{3 c}$ may seem to be a strange measure, but, in fact, much work has been done in the past to estimate such a lower bound. Calculation models and simulation programs based on "hydraulic" flow assumptions very often reports this number. The author, however, means that measure $\Phi_{3 c}$ is of little interest, and that its use is "dangerous" because it, in many cases, seriously underestimates the "real" evacuation time.

Category 4. These measures are directly related to $X_{i}(t)$.

$\Phi_{4 a}=$ Prob. distr. of $X_{i}(t)$

$\Phi_{4 b}=E X_{i}(t)$

$\Phi_{4 c}=$ Percentiles of $X_{i}(t)$

$\Phi_{4 d}=E Y_{i}$

$\Phi_{4 e}=E L_{i}$ 
$\Phi_{4 f}=E I_{i}$

$\Phi_{4 g}=E \bar{X}_{i}(\gamma)$

$\Phi_{4 h}=E X_{i}^{\max }$

Even if queueing may seem to be of minor importance, several sources claim that queueing is in itself dangerous, maybe causing people to crush each other, giving rise to panic, etc. Therefore, we choose to describe an evacuation system also by some queueing measures. The measures of this category may be related to the concept of "level of service", used by traffic planners. A discussion about these measures may be found in Løvås [9].

Category 5. These measures are related to the average redundancy number $F(t, s)$.

$\Phi_{5 a}=$ Prob. distr. of $F(t, s)$

$\Phi_{5 b}=E F(t, s)$

For the persons in the building at time $t$, it is important that there exist safe paths out of the building. Measure $\Phi_{5 b}$ gives information about the average number of safe independent paths for the persons in the building. This number will normally fall as $t$ grows, i.e. the situation will be worse for the persons, since the accident develops and make some evacuation routes "less attractive". If $E F(t, 0)$ equals zero, then the $E(K-M(t))$ persons in the building have a serious problem, since there are no more safe routes to follow. Then, it is interesting to know $E F(t, s)$ to see if there are any paths which are safe at level $s$. Measures $\Phi_{5}$. are well suited to tell whether the ES has high enough redundancy or not.

\section{QUANTITATIVE PERFORMANCE REQUIREMENTS}

For each relevant PM one has to define an acceptance criteria for the ES in question. This is a management process, and its outcome depends highly on the safety philosopy of the persons in charge. However, the QPRs must be expressed in measurable terms, relative to a welldefined PM. Typical examples of QPRs may therefore be:

- No more than $2 \%$ fatalities in the worst case scenario (ref. to $\Phi_{1}$ ).

- After 10 minutes, at least $95 \%$ of the building occupants should be safe (ref. to $\Phi_{2 b}$ ).

If the PMs indicate that the QPRs will (or may) not be met, then some corrective actions are needed to improve the $\mathrm{ES}$ performance. A list of recommended actions is given in Løvås [9].

\section{PERFORMANCE EVALUATIONS}

How can the PMs be evaluated? Several solution methods have been proposed in recent years, either analytical or based on simulations. An overview of different evacuation models may be found in [3] and [7]. 
Analytical solutions are hardly obtainable, and only for the "simplest" among the measures. For example, measure $\Phi_{3 c}$ may be studied by hydraulic flow models, whereas measure $\Phi_{1 a}$ or $\Phi_{4 a}$ can not be found in such a simple manner. Most of the analytical models presented in the literature are based on many quite unrealistic assumptions, not incorporating the extremely important aspects of human behaviour in a proper manner. Few of the analytical models include the accident, and most of them are made to optimize the routing of evacuees under highly idealized conditions. The author is therefore willing to conclude that such analytical models are of primarily academic interest, and not a very useful tool to fire safety personnel.

A survey conducted by Kostreva et al. [5] concluded that fairly extensive work has been done on the simulation of egress from a building on fire. All the models that they looked into included one or more of the following components: i) A network description of the building, ii) a set of heuristics for determining evacuee decisions, iii) a quantity that the evacuees are interested in minimizing, iv) input from a separate simulation that keeps track of the progress of the fire and smoke propagation and $v$ ) an algoritm. These tools differ in complexity, input requirements, underlying assumptions, and specific application areas. An overview of some existing methods has been given by Watts [12] and Lardeux [6], and a discussion about different models has been presented by L $\varnothing$ vås [7]. Two examples of simulation programs are documented by Fahy [4] and Ozel [11]. It is not within the scope of this paper to go into all details about any simulation program, but, since the author is involved in the development of EVACSIM, a short description of EvACSIM will be given below,

\section{Performance Evaluations with EvacsIM}

Evacsim is an evacuation simulation program under development in Norway and France. EvACSIM is presented in [2] and [3], and the program is described in detail in [13]. For additional information about EvaCsIM, see [14].

Data Input. The Evacsim user must describe the evacuation system in detail, using the Graphical Interface (GI) module of the program, presently running on SUN Sparc Stations. The building is modelled as the graph $G$, with nodes and links, represented by different icons in the GI. Values to node and link attributes may be given directly in associated windows which are opened and closed with the mouse. Examples of such attributes are initial population and capacity of a node, and width and length of a link. Links are bidirectional, but the user may indicate a preferrable direction of movement if escapeway marking signs are used. It is possible to load background pictures in standard CAD format.

Evacsim simulates the evacuation process on a microscopic level, in the sense that each person is treated as a separate "flow object". Some personal attributes are sampled from special distributions given by the user, while others are taken from an internal database where e.g. behavioural characteristics, obtained from literature reviews, are stored. The EvACSIM user must give the distribution of (uncongested) walking speed, fa- 
miliarity with the building, etc. Each person carries a lot of result-related variables, e.g. the path of movement, the doses of several types of accident exposure, etc. The persons has an attribute telling which exit the person is heading for. Initially, this attribute may be given, it may be random, or it may be the closest exit. During the evacuation process, the attribute may change if accident effects or congestion causes "trouble".

The accident scenario is represented as a dynamic scenario, specifying time and type of changes in accident effects in each node. The scenario may, and often will, be the output of a separate study with programs designed to simulate fire development and smoke flow.

The Evacuation Process. Evacsim performs a process-oriented discrete-event simulation, using the QNAP2-tool (Queueing Network Analyzing Package). This means that each person is followed closely and that critical events in his movement process are identified. These events are then scheduled into an event calender, and the program continues by handling the most imminent event on the event calender, corresponding to an event (activ$\mathrm{ity} /$ movement) for another person or a change in the accident scenario. Evacsim performs $R$ replications of the simulation from the same initial state (i.e. the same building topology, the same initial personnel localization, the same accident scenario). On each replication, values to the personal attributes are sampled from the appropriate distributions, and $K$ individual movement processes are "started". Each movement process is stochastic, e.g. because the persons are allowed to make "random" choices among alternative routes. Process $k$ obviously interacts with the other $K-1$ processes. Each replication is finished when all of the $K$ movement processes have stopped or when the maximum time $T$ is reached.

Evacsim includes several modules describing separate elements of the evacuation process. Four of the most important are: Initial Responce, Route Choice, Pedestrian Movement, Accident Effects. They are shortly described below.

The Initial Responce module decribes the human behaviour in the initial phase of the scenario. Its main objective is to estimate the time needed by each evacuee before the evacuation movement is initiated. Fire drills and results in the literature suggests that, in many cases, this initial phase makes a significant contribution to the evacuation time. See [13] for further details.

The Route Choice module describes the "random" mechanism governing the wayfinding behaviour of the evacuees. In EVACSIM, persons select their route "step by step", meaning that each person must make a choice in every node on his path. We define $p_{k}(i, j, \underline{X})$ as the probability that person $k$, with his personal attributes, will move from node $v_{i}$ to node $v_{j}$ when the whole system is in state $\underline{X}$. This probability is calculated for all possible choices, assuming that the persons follow a "perceived shortest path rule". This rule says that a person prefers a path which is perceived to be short. See [10] for further details.

The Pedestrian Movement module handles the movement process. Its main objective is to assign the actual walking speed to each person in each node, and to restrict the flow through the links. Detailed data exists in the literature, and this has formed the basis for this EvacsIM module. The walking speed depends on the personal walking ability and is 
a decreasing function of node occupancy. The link flow capacity is an increasing function of link-width. The movement module is well documented in [8].

The Accident Effects Module decribes how the evacuees are influenced by $\mathrm{CO}, \mathrm{CO}_{2}$, temperature and heat radiation. The accident effects are calculated based on results reported in the literature, typically stating the probability for fatal outcome at different exposure levels. EVACsIM models this in the following way: Each person is initially assigned tolerance levels to each exposure category. During his movement process, received doses of accident effects are accumulated and compared to the tolerance levels. If a tolerance level is passed, the person is assumed to be "dead" (or first unconscious), and reported as such in the simulation results. The module is well documented in [13].

Results. Statistical results are collected during the replicated simulations, and estimates to the performance measures $\Phi$ are presented as the main results. In addition, EvACSIM produces data which can be used to animate the evacuation process in the Graphical Interface. More detailed data is also collected to help the user to identify critical elements in the ES. In [9] an example is presented, showing several PMs computed by EvacsIM for a small building.

Use of EVACSIM. Tools like EvaCSIM have a wide application area, being able to simulate the evacuation process from almost all types of buildings. Evacsim has been successfully used to simulate evacuation from offshore oil production platforms and ships, and work has started to simulate evacuation from a hotel and a stadium.

Evacuation Simulators can be of great help to architects and civil engineers, especially in the critical early stages of the design process. Authorities responsible for the fire safety may use such tools to verify that performance goals are met. However, this depends on the predictive power of the simulators, and their validity.

\section{VALIDATION OF SIMULATION PROGRAMS}

The evacuation process is quite complex, and all simulation programs have to face the question about the validity of their results. In order to answer this question as neutral an well as possible, an evacuation model validation study is beeing planned in Norway, with support from the Royal Norwegian Research Council. The aim of this study is to look closely into many different simulation programs and present conclusions about their usefulness and predictive power.

The validation study will consist of three main parts: Modelling of historical accidents, modelling of fire drills which will be arranged and closely monitored, and a theoretical review of crucial assumptions and heuristics. The study will be managed by a neutral research institute (SINTEF) in Norway, with active participation from different model developers. All developers of evacuation simulation software are requested to seek information from [14]. 


\section{ACKNOWLEDGEMENTS}

Many thanks to Professors Terje Aven and Bent Natvig for helpful discussions over the presented topic; their experience with reliability theory, especially that of multistate systems and network flows, has been very inspiring. Thanks also to Jo Wiklund, Quasar Consultants, for useful ideas and critical comments. The author gratefully acknowledges the financial support of this work by the Royal Norwegian Council for Scientific and Industrial Research. Evacsim is developed by Quasar Consultants, Oslo, and Simulog, Paris, with finansial support from the French-Norwegian Foundation.

\section{REFERENCES}

[1] Bukowski, R.W.; Tanaka, T., "Toward the Goal of a Performance Fire Code", Fire and Materials, 15, pp. 175-180, 1991.

[2] Drager, K.H.; L $\varnothing$ vås, G.G.; Wiklund, J., "EvacsıM. A Comprehensive Evacuation Simulation Tool", Proc. of the 1992 Int. Emergency Management and Engineering Conference, Florida, April 1992. The Society for Computer Simulation (SCS). pp. 101-108.

[3] Drager, K.H.; L $\varnothing$ vås, G.G.; Wiklund, J., "Objectives of Modelling Evacuations from Buildings During Accidents", Journ. of Contingencies and Crisis Management, Vol. 1, No. 4, Blackwell, Oxford, UK, 1993.

[4] Fahy, R.F., "EXIT89: An Evacuation Model for High-Rise Buildings", Fire Safety Science-Proc. of the Third Int. Symp., Elsevier, London, pp. 815-823, 1991.

[5] Kostreva, M.M.; Wiecek, M.M.; Getachew, T., "Optimization Models in Fire Egress Analysis for Residental Buildings", Fire Safety Science-Proc. of the Third Int. Symp., Elsevier, London, pp. 805-814, 1991.

[6] Lardeux, E., "Crowd Movement Models", In K.E.Petersen and B.Rasmussen (eds.) Safety and Reliability '92, Elsevier, London, pp. 308-319, 1992.

[7] Løvås, G.G.; Wiklund, J.; Drager, K.H., "Evacuation Models and Objectives", In J.Sullivan (ed.) Proc. of the International Emergency Management and Engineering Conference, March 1993, Arlington, VA. The Society for Computer Simulation. pp. 91-100.

[8] Løvås, G.G., "Modeling and Simulation of Pedestrian Traffic Flow", To appear in Journal of Transportation Research, Part B.

[9] Løvås, G.G. "On Performance Measures for Evacuation Systems", To appear in the European Journal of Operations Research.

[10] Løvås, G.G. "Wayfinding in a Labyrinth", In review. 
[11] Ozel, F., "Simulation modeling of human behavior in buildings", Simulation, 58, 6, pp.377-384, 1992.

[12] Watts, J.M., "Computer Models for Evacuation Analysis", Fire Safety Journal, 12, 237-245, 1987.

[13] Wiklund, J.; Løvås, G.G., Functional Description of EvaCsIM, Quasar Report, 1993.

[14] Information about Evacsim is obtainable from: Quasar Consultants, Harbitzalleen 12, N-0275 Oslo, Norway. 